\title{
Precise Circadian Clocks in Prokaryotic Cyanobacteria
}

\section{Carl Hirschie Johnson*}

Department of Biological Sciences, Vanderbilt University, Nashville, TN 37235, USA

\begin{abstract}
Prokaryotic cyanobacteria express robust circadian (daily) rhythms under the control of a timing mechanism that is independent of the cell division cycle. This biological clock orchestrates global regulation of gene expression and controls the timing of cell division. Proteins that may be involved in input pathways have been identified. Mutational screening has identified three clock genes that are organized as a gene cluster. The structure of cyanobacterial clock proteins, their phosphorylation, and regulation is described. A new model for the core clockwork in cyanobacteria proposes that rhythmic changes in the status of the chromosome underlie the rhythms of gene expression. Mixed-strain experiments demonstrate that this timekeeper confers adaptive value when different strains compete against each other.
\end{abstract}

\section{Background: prokaryotes join the circadian club}

Before 1985, it was believed that circadian programs were exclusively found among eukaryotes (Johnson et al., 1996). The conclusion that only eukaryotes have circadian oscillators seemed reasonable, because it was assumed that an endogenous timekeeper with a period close to 24 $\mathrm{h}$ would not be useful to organisms that divide more rapidly than once every $24 \mathrm{~h}$, as do many prokaryotes (Pittendrigh, 1993). However, two reports in 1986 on circadian rhythms in nitrogen fixing cyanobacteria overturned that confident assumption (Grobbelaar et al., 1986; Mitsui et al., 1986). Our laboratory and those of our collaborators-Drs. Takao Kondo, Susan Golden, and Masahiro Ishiura-extended the studies on circadian programming to cyanobacteria that were genetically malleable (Johnson et al., 1996). Our first studies used a strain of Synechococcus elongatus PCC 7942 (previously named Synechococcus sp. strain PCC 7942) transformed with a luminescence reporter construct which is the fusion of the $p s b A /$ promoter with a bacterial luciferase cassette ( $p s b A / p:: / u x A B$; Kondo et al., 1993). This strain of cyanobacteria is notable because of the ease in which exogenous DNA can be transformed and homologously recombined into the chromosome (Andersson et al., 2000). Because the circadian clock turns this promoter on and off rhythmically, the reporter strain of $S$. elongatus glows rhythmically.

That choice of strain and promoter was due to the fact that Dr. Golden's laboratory had made this reporter strain for a completely different project. In retrospect, the choice

*For correspondence. Email carl.h.johnson@vanderbilt.edu. was a stroke of luck. Subsequent experiments using other strains/species of cyanobacteria have found rhythms (Aoki et al., 1997), but the reporters in those strains are not bright. And even in S. elongatus, other promoters do not show such a robust rhythm of luminescence (see Class 3-5 rhythms in Figure 1- $p s b A / p$ is a strong Class 1 promoter; Liu et al., 1995). The combination of the $p s b A / p:: / u x A B$ reporter and the $S$. elongatus strain remains one of the most robustly rhythmic combinations in cyanobacteria, even after ten years of intensive research. Dr. Kondo was able to exploit the bright and robust luminescence rhythm by designing clever apparatuses to monitor the rhythms. For liquid cultures, Dr. Kondo enlisted and modified the automated photomultiplier apparatus that was originally designed for endogenously bioluminescent algae (Kondo et al., 1993). For monitoring colonies, Drs. Kondo and Ishiura discovered that the rhythms of single colonies could be tracked with a CCD camera (Kondo and Ishiura, 1994).

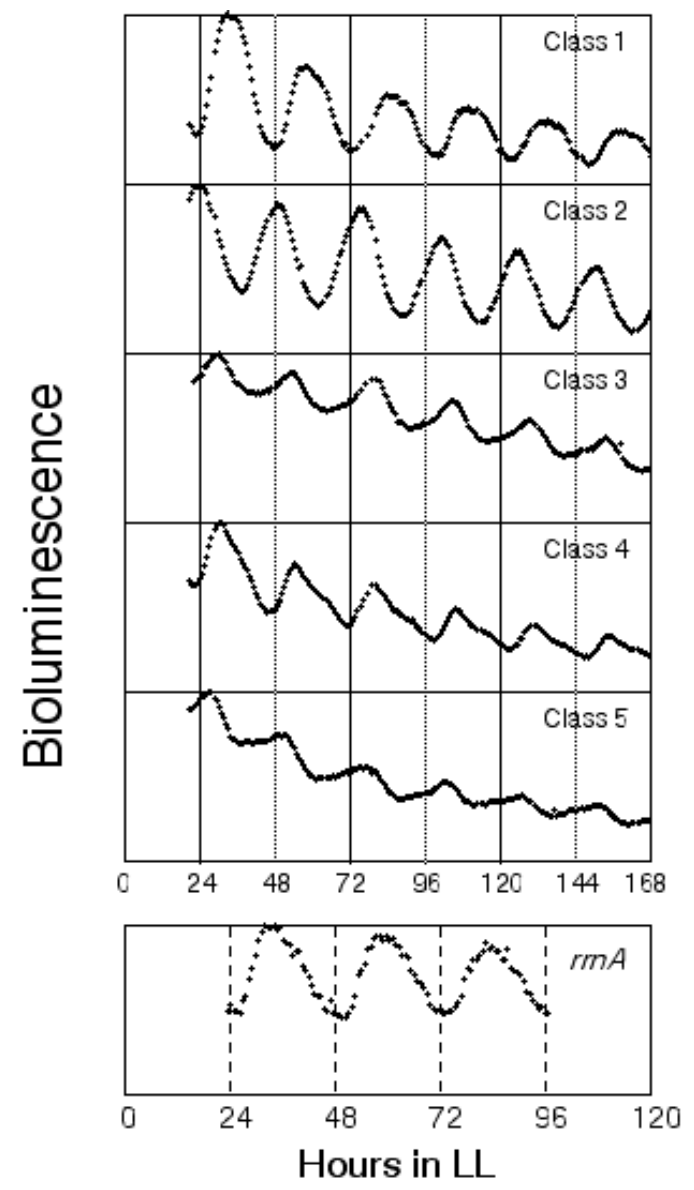

Figure 1. Global circadian regulation of promoter activities in S. elongatus. Upper five panels: representative traces of various classes of rhythmic waveforms resulting from the promoter trap experiment. Promoter activity is measured as bioluminescence. Lower panel: promoter activity of the rRNA gene, rrnA. Modified from Liu et al., 1995. 
Dr. Kondo used those observations to design an innovative turntable/CCD camera apparatus for mutant screening of single colonies, enabling the simultaneous screening of up to 12,000 colonies in a single experiment! (Kondo et al., 1994a).

The coincidence of good fortune, clever ideas, and hard work has transformed the $S$. elongatus system into one of the best characterized circadian clock systems, even though it is the newest comer to molecular clock analyses; only sixteen years ago, practically no one believed that prokaryotes were capable of circadian rhythmicity (Johnson et al., 1996; Kippert, 1991). Therefore, the S. elongatus system has rapidly caught up with eukaryotic systems, and in some areas, it is leading the cutting edge-particularly in terms of structural aspects of clock proteins (Williams et al., 2002; Mori et al., 2002; Hayashi et al., 2003), and of the rigorous assessment of adaptive significance (Ouyang et al., 1998).

The issues of adaptive significance and occurrence of clocks in prokaryotes naturally lead to a consideration of evolutionary relationships. Later in this article, the genetics and function of the central clock gene cluster (kaiABC) will be discussed. These genes are widely distributed among cyanobacteria (Lorne et al., 2000). There are putative homologs of KaiC in other bacteria, especially archaebacteria, but no homologs to any of the Kai proteins have yet been discovered in eukaryotes (Johnson and Golden, 1999: DasSarma et al., 2001; Dvornyk et al., 2003). A genomic survey claimed that KaiC is a member of the bacterial RecA/DnaB family (Leipe et al., 2000). RecA is an ATP-dependent DNA recombinase, and DnaB is the replication fork helicase in bacteria. The kaiC gene of $S$. elongatus appears to be an internally duplicated version of a RecA/DnaB-like gene; it has two parts that are very similar to each other (Leipe et al., 2000; Iwasaki et al., 1999). In Eubacteria (cyanobacteria et al.), kaiC has only been found in the "double-domain" version, whereas in Archaea, both single-domain and double-domain versions of kaiC have been found. These observations have stimulated hypotheses for the evolutionary origins of the kai genes (Leipe et al., 2000; Dvornyk et al., 2003).

\section{Outputs}

A remarkable property of the circadian system in $S$. elongatus is that it globally regulates essentially all promoters in the genome, as shown by a sensitive and comprehensive "promoter trap" experiment (Liu et al., 1995). A promoterless luciferase gene set was randomly inserted throughout the genome; whenever the luciferase gene set inserted into a genomic locus that was correctly positioned and oriented to a promoter, luciferase was expressed and the colonies glowed. The luminescent patterns of 3000 independent colonies were analyzed. Unexpectedly, all of the glowing colonies displayed circadian rhythms with the same period (Liu et al., 1995). The pattern of the rhythmic expression differed among the promoters, both in terms of phasing and waveform, as shown in Figure 1. Figure 1 also shows that the promoter activity for a ribosomal RNA gene (rrnA) is likewise rhythmic. Apparently the cyanobacterial clock globally controls gene expression-in this case, the activity of promoters, and in a separate study of Synechococcus RF-1, of protein abundances (Huang et al., 1994). Moreover, when a heterologous E. coli promoter (conl/p) is tested in S. elongatus, it is not only transcribed, but it is rhythmically active (Katayama et al., 1999). A model for this global control will be presented below.

As seen in Figure 1, there are several "classes" of promoter activity patterns. In particular, several of the classes are phased differently. Because most of the promoters are phased into a Class 1 phase, the other phases are especially interesting. Class 3 phasing has not been reproducible from experiment to experiment, but there are several promoters that reliably express in the Class 2 phase, i.e., in anti-phase to Class 1 . This phasing means that expression will be largely in the night. One of the Class 2 genes that has been identified and characterized is purF, the rate-limiting enzyme of the purine biosynthetic pathway (Liu et al., 1996). An interesting feature of the PurF enzyme is that its activity is inhibited by oxygen. Its nighttime expression might be adaptive to avoid the oxygen generated by photosynthesis in the daytime (Liu et al., 1996). Another Class 2 promoter is that driving the expression of opcA, a gene that is involved in temporal control of pathways that generate reductants (Min and Golden, 2000).

There are other outputs besides gene expression of the cyanobacterial biological clock. In other cyanobacterial species there are rhythms of nitrogen fixation, amino acid uptake, and photosynthesis (Grobbelaar et al., 1986; Mitsui et al., 1986; Huang et al., 1994; Chen et al., 1991). S. elongatus is not capable of nitrogen fixation, but it also appears to have rhythms of photosynthetic capacity. There is a significant (but not very robust) rhythm of photosynthetic oxygen production that peaks in the subjective day (A. Dean and T. Mori, unpublished results). Probably related to the rhythm of photosynthesis is a rhythm of delayed chlorophyll fluorescence (Kondo et al., 1994b).

Finally, another output of the circadian clock in cyanobacteria is the timing of cell division. As mentioned above, it had been assumed that a circadian oscillator would not be useful to organisms that divide more rapidly than once every $24 \mathrm{~h}$ (Pittendrigh, 1993). We now recognize that these assumptions were based on fallacious "eukaryote-centric thinking" (Mori and Johnson, 2000: Johnson and Kondo, 2001). Cyanobacteria express robust circadian rhythms of gene expression when dividing with doubling times of 6-12 h (Mori et al., 1996: Kondo et al., 1997). They also express excellent rhythms when they are not dividing at all (Mori and Johnson, 2001a). Therefore, the circadian clock is independent of the cell division cycle in cyanobacteria-unfettered by the status of cell division, the circadian clock "keeps on ticking". However, the timing of cell division is not independent of the circadian clock; even when the cells are dividing rapidly, the circadian clock rhythmically slows the rate of cell division every night. Even in constant light, the rate of cell division is slowed in the early subjective night (Mori et al., 1996). Apparently, the circadian clockwork is well buffered and stable against significant changes of the intracellular milieu. Consequently, the circadian clockwork in S. elongatus gates cell division and gene expression, but its timing circuit is independent of the cell division cycle. 


\section{Inputs and Entrainment}

Both light/dark and temperature cycles can entrain cyanobacterial clocks. In a study of Synechococcus RF-1, light/dark cycles appear to be a more potent zeitgeber than temperature cycles (Lin et al., 1999). Which photopigments are involved in the entrainment by light/dark signals? A spectral study of the wavelengths of light that are effective phase-resetting cues would be useful. Light-induced phase shifting by the usual protocol (i.e., light pulses in a DD background) has been difficult to test in $S$. elongatus because the cells need to be in LL to allow robust circadian gene expression, and light pulses on an LL background have little phase-shifting efficacy. On the other hand, dark pulses on an LL background are very effective resetting agents of the $S$. elongatus clock (Kondo et al., 1993). We therefore designed a protocol to determine which wavelengths of light would be effective in reversing a dark-pulse-induced phase shift. In other words, a dark pulse was compared with a blue, green, yellow, or red light pulse. Those wavelengths that have the same phase-shifting effect as a dark pulse are therefore those to which the clock is "blind." Wavelengths that provide input to the clock would be those that appear similar to the constant light control. In an action spectroscopy test of this protocol, we found that both blue and red light were effective input signals (peaks at $440 \mathrm{~nm}$ and 600-650 nm; Kondo and Johnson, unpublished results). Moreover, long exposures to the photosynthesis inhibitor, Diuron, can mimic the effect of dark pulses (Katayama et al., 2003). Those observations suggest that photosynthetic electron transport could act as a clock entrainment input. However, the interpretation that photosynthesis can act as an input is premature, and better action spectrum data are required to answer this question.

A different strategy to identify input components used a transposon mutagenesis screen for disrupted phase resetting to a $5 \mathrm{~h}$ dark pulse (Katayama et al., 2003; Schmitz et al., 2000). This screen identified some period mutants, including two in the gene encoding an iron-sulfur protein named LdpA (Katayama et al., 2003). One mutant identified in that screen showed a reduced phase-shifting response to the dark pulse and was identified as a phytochromelike histidine protein kinase, called CikA (Schmitz et al., 2000). CikA can autophosphorylate but does not bind bilin in vivo, as expected for a phytochrome (Mutsuda et al., 2003). While CikA's phytochrome-like sequence implied its action as a photopigment, a surprising result is that CikA is required for response to phase-shifting temperature signals as well to light/dark signals (Mutsuda et al., 2003). It is important to note that while cikA mutant strains do not phase shift in response to $5 \mathrm{~h}$ dark pulses (Schmitz et al., 2000), it will entrain effectively to $12 \mathrm{~h}$ dark pulses. This result suggests that CikA does not mediate the only input pathway. Apparently, a variety of input signals can entrain the S. elongatus clock.

\section{Core mechanism: the kaiABC clock gene cluster}

In work that was primarily performed in Dr. Kondo's lab in Japan, the turntable/CCD apparatus was used with the luciferase reporter strain of $S$. elongatus in EMS mutagenesis screens to isolate more than 100 circadian mutants (Kondo et al., 1994a). Some of these mutants were rescued by the introduction of libraries of wild-type S. elongatus DNA. Those rescue experiments pinpointed a cluster of three adjacent genes, named kaiA, kaiB, and kaiC (Ishiura et al., 1998). The kaiABC cluster appears to be a clock-specific region of the chromosome in cyanobacteria because deletion of the entire cluster or of any one of the kai genes separately does not affect viability (in single-strain cultures), but causes arhythmicity. Promoters were found in the upstream regions of both the kaiA and kaiB genes. The kaiA promoter (kaiAp) gives rise to a monocistronic kaiA mRNA, whereas the kaiB promoter (kaiBCp) produces a dicistronic kaiBC mRNA (Ishiura et al., 1998).

As mentioned above, kaiC appears to be an internally duplicated version of a $r e c A / d n a B$-like gene; it has two parts that are very similar to each other (Iwasaki et al., 1999). In each half of the kaiC gene, there is a Walker A or "P-loop" motif. This motif, [G or A]XXXXGK[T or S], is often a ATP/ GTP nucleotide binding region. Indeed, KaiC binds ATP, and when the Walker A motifs are mutated, the nucleotide binding is eliminated and rhythmicity is severely disrupted or abolished (Nishiwaki et al., 2000; Mori and Johnson, 2001b). Therefore, this motif is essential in both parts of the KaiC molecule for rhythmicity. As with eukaryotic clock proteins, interactions are important. KaiA, KaiB, and KaiC interact with each other (Iwasaki et al., 1999: Xu et al., 1999; Taniguchi et al., 2001). KaiC also interacts with a histidine kinase, SasA, that is crucial for maintaining a high amplitude, robust rhythm of gene expression (Iwasaki et al., 2000). These interactions appear to lead to the formation of complexes in vivo (Kageyama et al., 2003). There is also evidence for the association of a population of KaiB and KaiC molecules with membranes (Kitayama et al., 2003).

In some of the first structural work on clock proteins from any system, an NMR analysis indicated that the $\mathrm{N}$-terminal domain of KaiA is a pseudo-receiver domain (Williams et al., 2002). In work from our lab, electron microscopy coupled with analytical ultracentrifugation, chromatography, and native gel electrophoresis has demonstrated that purified KaiC will form ATP-dependent hexameric ring complexes in vitro, as shown in Figure 2 (Mori et al., 2002; Hayashi et al., 2003). These hexameric structures are similar to those found in DnaB and RecA and further supports the sequence analyses that placed KaiC in the RecA/DnaB superfamily (Leipe et al., 2000). Moreover, these KaiC hexamers bind DNA (Mori et al, 2002).

Some features of kai gene regulation are reminiscent of the regulation of eukaryotic clock genes. There are rhythms in the abundance of kaiA and kaiBC transcripts (Ishiura et al., 1998) and the KaiB and KaiC proteins (Xu et al., 2000; Iwasaki et al., 2002). Continuous overexpression of KaiC repressed the kaiBC promoter, suggesting negative feedback of KaiC on its promoter in an analogous fashion to the case for eukaryotic clock proteins (Ishiura et al., 1998). Pulsatile expression of the kaiC gene within physiological levels from an inducible promoter resets the phase of the rhythms (Ishiura et al., 1998; Xu et al., 2000). The observation that KaiC is rhythmically abundant and that pulses of KaiC expression elicit phase resetting led to the hypothesis that KaiC turnover might be an important determinant of clock period and phase (Xu et al., 2000). 

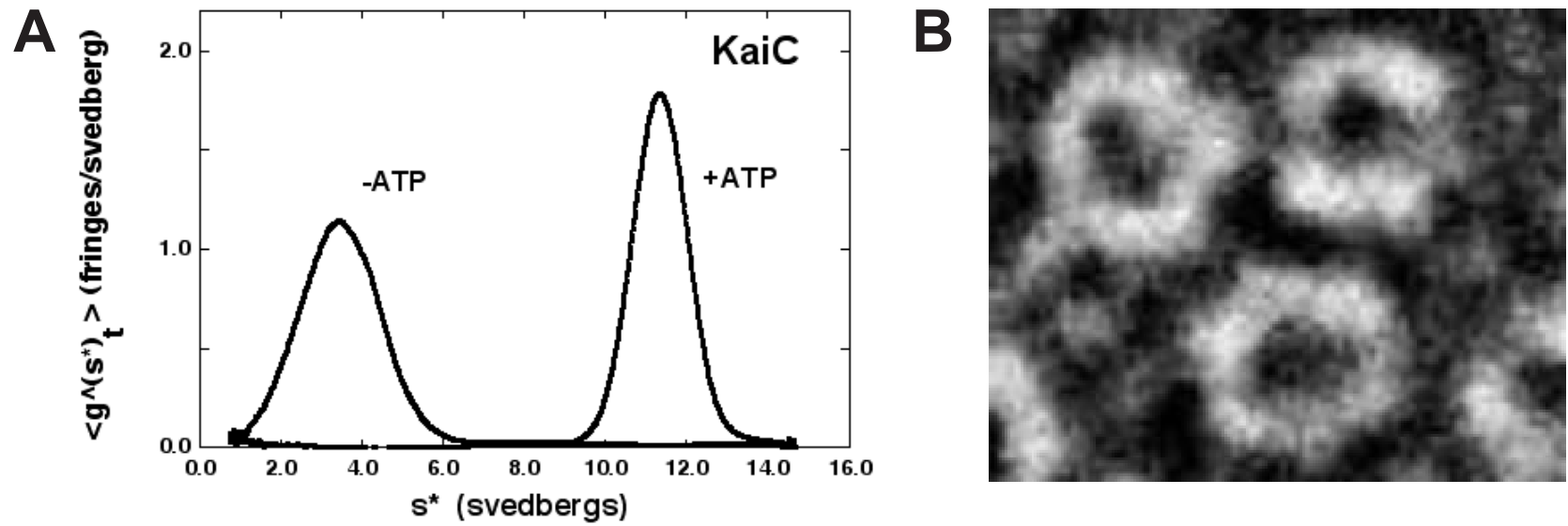

Figure 2. ATP-dependent protein complexes formed by the KaiC protein from Synechococcus. A. As determined by analytical ultracentrifugation, KaiC forms complexes in the presence of ATP that are hexamers (sedimentation coefficient of about $11.5 \mathrm{~S}$ ). In the absence of ATP, KaiC is monomeric (sedimentation coefficient of about 3.6 S). B. Negatively stained hexameric ring complexes viewed by electron microscopy. Modified from Mori et al., 2002.

This hypothesis was tested in a recent publication that found support for KaiC degradation rate as a partial determinant of period (Xu et al., 2003).

KaiC exists in phosphorylated forms in vivo (Iwasaki et al., 2002), suggesting another similarity to the posttranslational control of eukaryotic clock proteins. The ratio of phosphorylated to non-phosphorylated KaiC is correlated with the period at which the clock runs (Xu et al., 2003). Unlike some eukaryotic systems, however, the extent of KaiC phosphorylation is not directly correlated with KaiC degradation (Xu et al., 2003). Purified recombinant KaiC can autophosphorylate in vitro (Nishiwaki et al., 2000; Iwasaki et al., 2002; Williams et al., 2002). This autophosphorylation is stimulated in vitro by KaiA (Iwasaki et al., 2002; Williams et al., 2002). On the other hand, KaiB antagonizes the effects of KaiA on KaiC autophosphorylation (Williams et al., 2002; Kitayama et al., 2003). KaiC will also de-phosphorylate in vitro, implying that KaiC will continuously turn over its phosphorylation status in the presence of ATP (Xu et al., 2003). We found that dephosphorylation of KaiC in vitro is inhibited by KaiA, and this effect of KaiA is also antagonized by KaiB (Xu et al., 2003). It is possible that the effects of KaiA and KaiB on KaiC dephosphorylation in vitro can account for the results of KaiA and KaiB on KaiC autophosphorylation (Xu et al., 2003)-e.g., perhaps the apparent KaiA stimulation of KaiC autophosphorylation is due to an inhibition of dephosphorylation (Iwasaki et al., 2002; Williams et al., 2002; Kitayama et al., 2003). However, for the time being, we will adopt the interpretation that KaiA both stimulates KaiC autophosphorylation and inhibits its dephosphorylation (Figure 3 ). The in vitro results are relevant to events happening within cells-in vivo, KaiA stabilizes KaiC in the phosphorylated form, and KaiB antagonizes the effect of KaiA (Iwasaki et al., 2002; Xu et al., 2003). Moreover, an extensive PCR mutagenesis of KaiA showed that the vast majority of KaiA mutations slowed the period of the clock (Nishimura et al., 2002). The role of KaiA on stabilizing KaiC phosphorylation and the KaiA mutant analysis implies that phosphorylated KaiC is needed to speed the clock.

Not only is KaiC phosphorylated in vivo, but the proportion of phospho-KaiC to non-phospho-KaiC oscillates over the circadian cycle (Iwasaki et al., 2002). Our lab had previously reported that $\mathrm{KaiC}$ is rhythmically abundant (Xu et al., 2000). Based on the work of Iwasaki and coworkers (Iwasaki et al., 2002), we now realize that the multiple KaiC bands we observed in our previous time course study of wild-type cells (Xu et al., 2000) are indicative of rhythms of KaiC phosphorylation in which P-KaiC predominates in the subjective night (upper bands). The abundance of nonP-KaiC is also rhythmic, although the nonP-KaiC band was obscured in our previous investigation by a crossreacting antigen (Xu et al., 2000). The rhythmic band that we identified as KaiC in our immunoblots is phospho-KaiC, and non-phospho-KaiC on our blots is a faster mobility band that co-migrates with a cross-reacting antigen ( $\mathrm{Xu}$ et al., 2000). Therefore, our results concur with those of Iwasaki and coworkers in demonstrating a circadian rhythm of the ratio of $\mathrm{P}-\mathrm{KaiC}$ to nonP-KaiC in vivo (Iwasaki et al., 2002; Xu et al., 2003).

Figure 3 summarizes some of these data. The kaiA, $B$, and $C$ genes are rhythmically transcribed. Probably the mRNAs are rhythmically translated, but KaiA is relatively stable so that its abundance is not rhythmic (Xu et al., 2000; Kitayama et al., 2002). The abundances of KaiB and KaiC are rhythmic (Xu et al., 2000; Kitayama et al., 2002). The Kai proteins interact and possibly form a complex in vivo (Kageyama et al., 2003). KaiC autophosphorylates and forms a hexamer that may form the core of a complex (Mori et al., 2002; Hayashi et al., 2003). The ratio of phosphoKaiC to non-phospho-KaiC is regulated by KaiA, which stabilizes the phosphorylated form of KaiC, and by KaiB, which antagonizes the actions of KaiA (Iwasaki et al., 2002; Williams et al., 2002; Xu et al., 2003). The extent of KaiC phosphorylation is correlated with clock speed in vivo (Figure 3; Xu et al., 2003).

\section{Core mechanism: a new model-the "oscilloid"}

Taken together with analogous data from eukaryotic clocks, the results from studies up to the year 2000 had been used to support a model wherein there is negative feedback control of kaiBC expression by the KaiC protein acting upon kaiBCp to generate a circadian oscillation in which 


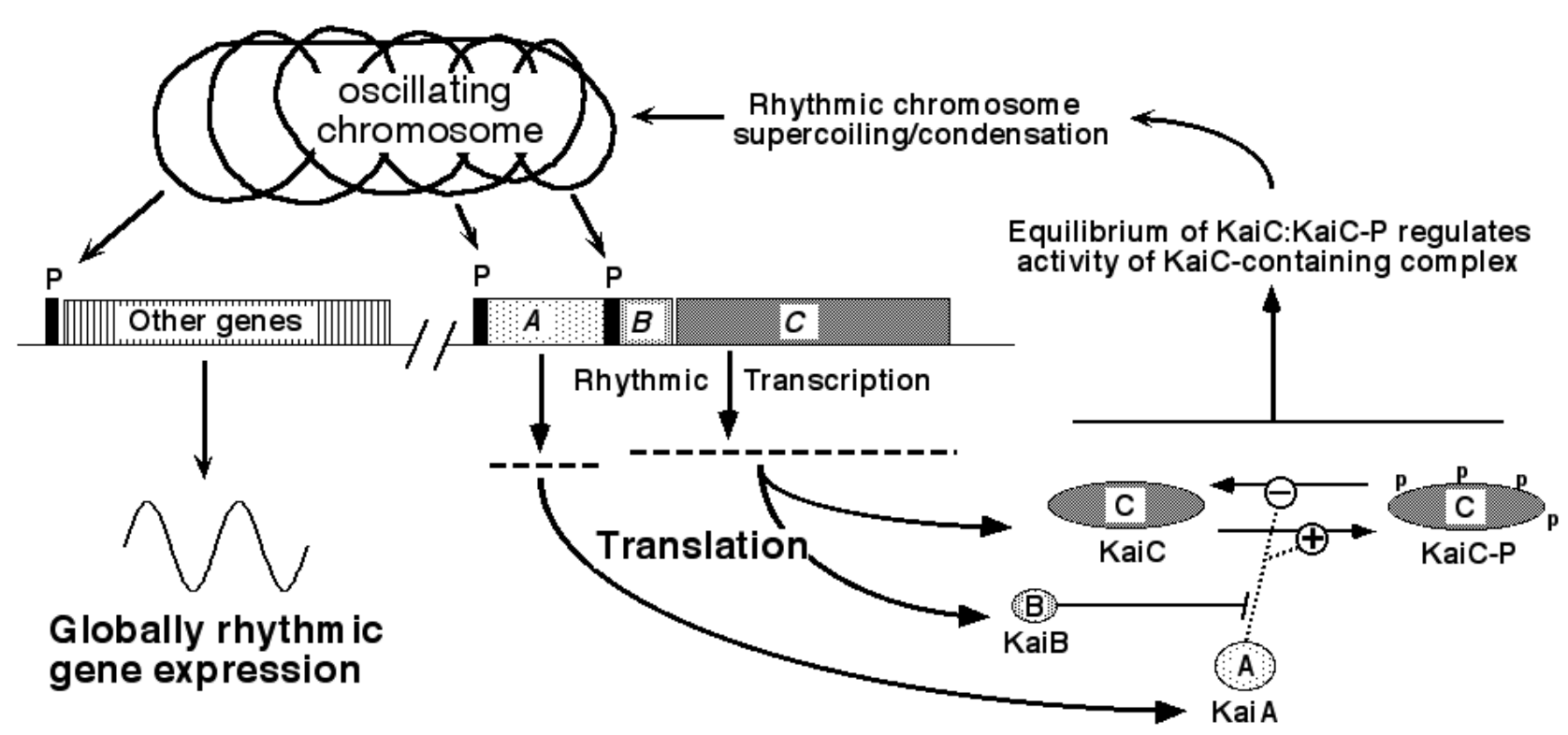

Figure 3. "Oscilloid" model for the circadian oscillator of S. elongatus. KaiA, KaiB, and KaiC are transcribed and translated from the kaiABC cluster using two promoters: kaiAp and kaiBCp. KaiA promotes the phosphorylation of KaiC and inhibits its dephosphorylation, while KaiB antagonizes the actions of KaiA. KaiC phosphorylation is coincident with the formation of a KaiC-containing complex that mediates rhythmic and global changes in the status of the chromosome. These changes in chromosomal status influence the transcriptional activity of all promoters in the chromosome so that there are global circadian changes in gene expression. "Other genes" includes heterologous genes (and heterologous promoters such as trcp). Modified from Xu et al., 2003.

the stability of KaiC is influenced by its phosphorylation status and thereby modulates period length (Ishiura et al., 1998; Nishiwaki et al. 2000). This model implies that the biochemistry of the clockwork in cyanobacteria is similar to that in eukaryotes. One of the predictions of that model is that the circadian feedback loop is dependent upon proper kaiBCp function. An ancillary expectation was that global gene expression might be mediated by changes in sigma subunits of RNA polymerase that would rhythmically turn on and off groups of genes. However, studies of sigma subunits in cyanobacteria have not yielded explanations for global regulation (Tsinoremas et al., 1996; Nair et al., 2002). Even more unexpectedly, we found that the kaiBC promoter is not specifically required for the circadian oscillator to operate in cyanobacteria-a non-specific heterologous promoter can drive kaiBC expression and obtain essentially wild-type circadian patterns (Xu et al., 2003; Y. Nakahira and T. Kondo, submitted).

Three lines of evidence led us to propose a new model for the fundamental mechanism of the clockwork in S. elongatus: (1) global circadian regulation of promoters, even heterologous (e.g., conl/p) promoters; (2) when $\mathrm{KaiB}$ and KaiC expression is driven from a non-specific heterologous promoter (trcp) instead of the endogenous kaiBC promoter, rhythmicity persists; and (3) the key clock protein KaiC binds DNA and is related to bacterial recombinases and helicases, implying that KaiC might act directly on DNA (Mori and Johnson, 2001b). These observations emboldened us to consider that KaiC might mediate both its own negative feedback regulation (Ishiura et al., 1998) and global regulation of the cyanobacterial genome (Liu et al., 1995) by orchestrating oscillations in the condensation and/or supercoiling status of the entire cyanobacterial chromosome. The chromosome of most bacteria is organized into a "nucleiod," which has a highly organized architecture based on condensation and coiling of DNA (Pettijohn, 1976; Trun and Marko, 1988). It is well known that changes in the local supercoiling status of DNA can affect the transcription rate of genes (Menzel and Gellert, 1987; Pruss and Drlica, 1989; Hsieh et al., 1991).

We therefore proposed that a fundamental component of the cyanobacterial clockwork is that the condensation/supercoiling status of the chromosome rhythmically changes such that it becomes an oscillating nucleoid, or "oscilloid" (Figure 3; Mori and Johnson, $2001 b$ ). There is already precedence for daily rhythms of supercoiling status in the chloroplast chromosome of the eukaryotic alga Chlamydomonas (Salvador et al., 1998). In cyanobacteria, we postulate that these condensation/ supercoiling oscillations promote rhythmic modulation of the transcription rates of all genes, accounting for global regulation of gene expression. Therefore, gene-specific ciselements that mediate rhythmic gene expression might be (at least partially) responsive to chromosomal status rather than exclusively to trans factors. In addition, heterologous promoters (or trcp::kaiC constructs) that are integrated into the chromosome are driven rhythmically since they are also subjected to the oscillating chromosomal status. A minor proportion of the genes oscillate in the opposite phase relationship (e.g., purF) to the majority of the genes, and this might be due to differential responses in different domains of the chromosome, or to the fact that different promoters react differently to negative vs. positive supercoiling (Drlica, 1992). Finally, we suggest that KaiC (or most likely, a KaiC-containing complex) is a key player in regulating these changes of chromosomal status, and that the phosphorylation status of KaiC is important in the 


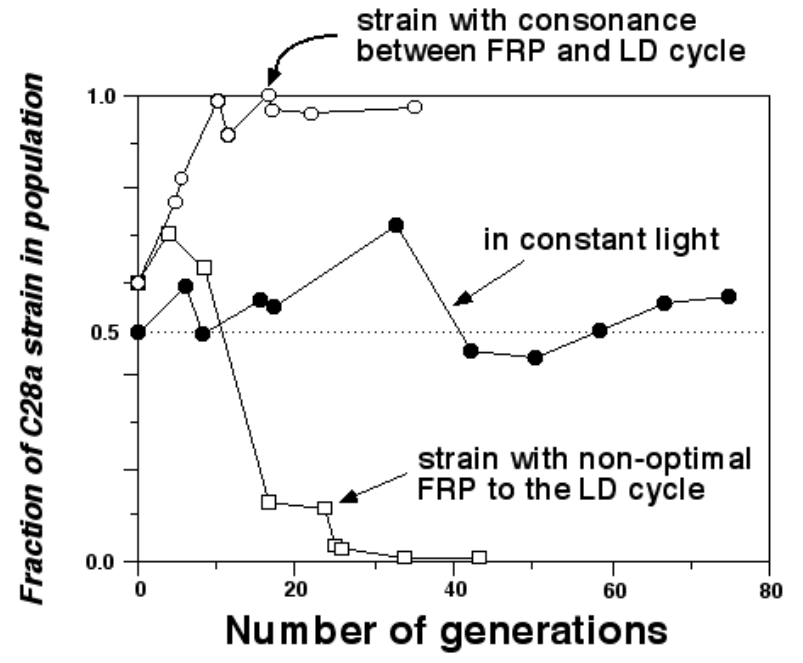

Figure 4. Competition of circadian strains in different light/dark cycles. Different strains of cyanobacteria were mixed and grown in competition under different light/dark cycles. The strain whose endogenous free-running period most closely matched that of the environmental light/dark cycle was able to out-compete strains with a non-optimal period. In constant light (non-selective conditions), all the strains were able to maintain their initial fraction in the population. FRP = free-running period, $L D=$ light/dark cycle. These data show the competition between a 30-h mutant (C28a) and wildtype (FRP = $24 \mathrm{~h}$ ). The upper trace is on a 30-h LD cycle, the middle trace is from constant light, and the lower trace is on a 24-h LD cycle. Ordinate, fraction of C28a mutant in the mixed population (modified from Ouyang et al. 1998).

regulation of this complex's activity (Figure 3; Xu et al., 2003).

\section{Does this clock enhance fitness?}

The study of circadian rhythms has been dedicated to the proposition that temporal programs regulated by these clocks are adaptive. However, prior to 1998 there had been no direct, definitive tests for whether circadian clocks enhance fitness in any organism. We performed the first rigorous test of the adaptive significance of circadian programs by using competition experiments between different strains of the cyanobacterium S. elongatus (Ouyang et al., 1998). For asexual microbes such as $S$. elongatus, differential growth of one strain under competition with other strains is a good measure of reproductive fitness. In pure culture, the strains grew at about the same rate in constant light and in light/dark cycles, so there did not appear to be a significant advantage or disadvantage to having different circadian periods when the strains were grown individually. The fitness test was to mix different strains together and to grow them in competition to determine if the composition of the population changes as a function of time. The cultures were diluted at intervals to allow growth to continue.

In experiments in which wild-type strains were competed with arhythmic strains, the arhythmic strains were rapidly defeated by wild-type strains in light/dark cycles, but under competition in constant light, the arhythmic strains grew as well (and sometimes better than!) wild-type strains (Y. Ouyang and M. Woelfle, unpublished observations). These results indicate that having a circadian clock improves fitness of cyanobacterial cells in a selective environment (i.e.,a light/dark cycle), but not in a non-selective environment (i.e., constant light).

Different period mutants were used to answer the question, "does having a period that is similar to the period of the environmental cycle enhance fitness?" The circadian phenotypes of the strains used had free-running periods of about 22 h, $25 \mathrm{~h}$ (wild-type), and $30 \mathrm{~h}$. When each of the strains was mixed with another strain and grown together in competition, a pattern emerged that depended upon the frequency of the light/dark cycle and the circadian period. When grown on a $22-\mathrm{h}$ cycle (11 h light, $11 \mathrm{~h}$ dark), the $22 \mathrm{~h}$-period mutant became the dominant cell type in the mixed cultures. On a 30 -h cycle (15 h light, 15 h dark), the 30 h-period mutant ("C28a") could defeat either wild type or the $22 \mathrm{~h}$-period mutant. On a "normal" $24 \mathrm{~h}$ cycle $(12 \mathrm{~h}$ light, $12 \mathrm{~h}$ dark), the wild type strain could overgrow either mutant (Ouyang et al., 1998). Figure 4 shows results from the competition between wild-type and the $30 \mathrm{~h}$-period mutant C28a. Strains in which other clock genes were mutated showed the same effect (K. Phanvijhitsiri and M. Woelfle, unpublished data).

Clearly, the strain whose period most closely matched that of the light/dark cycle eliminated the competitor. Under a non-selective condition (in this case, constant light), each strain was able to maintain itself in the mixed cultures. Because the mutant strains could defeat the wild-type strain in LD cycles in which the periods are similar to their endogenous periods, the differential effects that were observed are likely to result from the differences in the circadian clock. A genetic test was also performed to demonstrate that the clock gene mutation was specifically responsible for the differential effects in the competition experiment (Ouyang et al., 1998). This was the first rigorous demonstration in any organism of a fitness advantage conferred by a circadian system. What is the basis of this competitive advantage? The results of modeling studies favor the interpretation that the cyanobacteria rhythmically secrete an inhibitor (Gonze et al., 2002). This interpretation has not yet been experimentally confirmed.

\section{The significance of clock studies in cyanobacteria}

Research on circadian programming in cyanobacteria has overturned at least two dogmas-(1) that prokaryotes cannot have circadian clocks, and (2) that organisms cannot express circadian rhythms when they divide more rapidly than once per 24 hours. These investigations have also provided the first rigorous evidence for a fitness advantage conferred by circadian programming, and for global orchestration of gene expression. The investigation of its central mechanism has progressed rapidly within a short time, but this clockwork remains mysterious. We proposed herein a new model to account for global gene regulation and for the role of the essential clock protein KaiC by envisioning an oscillating chromosome, the oscilloid. If this model is found to be true, it will introduce a new way in which circadian gene expression is regulated by DNA structure. 


\section{Acknowledgements}

I thank Drs. Takao Kondo and Susan Golden for a productive and exciting collaboration; most of the work described herein is a product of our joint efforts. I am grateful for support from the National Science Foundation and the National Institutes of Health.

\section{References}

Andersson, C.A., Tsinoremas N.F., Shelton, J., Lebedeva, N.V., Yarrow, J., Min, H., and Golden, S.S. (2000). Application of bioluminescence to the study of circadian rhythms in cyanobacteria. Methods Enzymol. 305, 527-542.

Aoki, S., Kondo, T., Wada, H., and Ishiura, M. (1997). Circadian rhythm of the cyanobacterium Synechocystis sp. PCC 6803 in the dark. J. Bacteriology 179, 57515755.

Chen, T.-H., Chen, T.-L., Huang, L.-M. and Huang, T.C. (1991). Circadian rhythm in amino acid uptake by Synechococcus RF-1. Plant Physiol. 97, 55-59.

DasSarma, S., Kennedy, S.P., Berquist, B., Ng, W.V., Baliga, N.S., Spudich, J.L., Krebs, M.P., Eisen, J.A., Johnson, C.H., and Hood, L. (2001). Genomic perspective on the photobiology of Halobacterium species NRC-1, a phototrophic, phototactic, and UV-tolerant haloarchaeon. Photosynthesis Res. 70, 3-17.

Dvornyk, V., Vinogradova, O., and Nevo, E. (2002). Origin and evolution of circadian clock genes in prokaryotes. Proc Natl Acad Sci USA 100, 2495-2500.

Drilca K. (1992). Control of bacterial DNA supercoiling. Mol Microbiol 6, 425-433.

Gonze, D., Roussel, M.R., Goldbeter, A. (2002). A model for the enhancement of fitness in cyanobacteria based on resonance of a circadian oscillator with the external light-dark cycle. J. Theor. Biol. 214, 577-597.

Grobbelaar, N., Huang, T.-C., Lin, H.Y., and Chow, T.J. (1986). Dinitrogen-fixing endogenous rhythm in Synechococcus RF-1. FEMS Microbiol. Lett. 37, 173177.

Hayashi, F., Suzuki, H., Iwase, R., Uzumaki, T., Miyake, A., Shen, J.-R., Imada, K., Furukawa, Y., Yonekura, K., Namba, K., and Ishiura, M. (2003). ATP-induced hexameric ring structure of the cyanobacterial circadian clock protein KaiC. Genes to Cells 8, 287-296.

Hsieh, L.S., Rouviere-Yaniv, J., Drlica, K. (1991). Bacterial DNA supercoiling and $[A T P] /[A D P]$ ratio: changes associated with salt shock. J Bacteriol 173, 3914-3917.

Huang, T.-C., Chen, H.-M., Pen, S.-Y., and Chen, T.-H. (1994). Biological clock in the prokaryote Synechococcus RF-1. Planta 193, 131-136.

Ishiura, M., Kutsuna, S., Aoki, S., Iwasaki, H., Andersson, C.R., Tanabe, A., Golden, S.S., Johnson C.H., Kondo, T. (1998). Expression of a gene cluster kaiABC as a circadian feedback process in cyanobacteria. Science 281, 1519-1523.

Iwasaki, H., Taniguchi, Y., Kondo, T., Ishiura, M. (1999). Physical interactions among circadian clock proteins, KaiA, KaiB and KaiC, in Cyanobacteria. EMBO J. 18, 1137-45.

Iwasaki, H., Williams, S.B., Kitayama, Y., Ishiura, M.,
Golden, S.S. and Kondo, T. (2000). A KaiC-interacting sensory histidine kinase, SasA, necessary to sustain robust circadian oscillation in cyanobacteria. Cell 101, 223-233.

Iwasaki, H., Nishiwaki, T., Kitayama, Y., Nakajima, M., and Kondo, T. (2002). KaiA-stimulated KaiC phosphorylation in circadian timing loops in cyanobacteria. Proc. Natl. Acad. Sci. USA 99, 15788-15793.

Johnson, C.H., Golden, S.S., Ishiura, M., Kondo, T. (1996). Circadian clocks in prokaryotes. Mol. Microbiol. 21, 511.

Johnson, C.H. and Golden, S.S. (1999). Circadian programs in cyanobacteria: adaptiveness and mechanism. Annu. Rev. Microbiol. 53, 389-409.

Johnson, C.H. and Kondo, T. (2001). Circadian rhythms in unicellular organisms. In: Handbook of Behavioral Neurobiology, Chapter 3. Plenum Press, p. 61-77.

Kageyama, H., Kondo, T. and Iwasaki, H. (2003). Circadian formation of clock protein complexes by KaiA, KaiB, KaiC, and SasA in cyanobacteria. J. Biol. Chem. 278, 2388-2395.

Katayama, M., Tsinoremas, N.F., Kondo, T., and Golden, S.S. (1999). cpmA, a gene involved in an output pathway of the cyanobacterial circadian system. J. Bacteriol., 181, 3516-3524.

Katayama, M., Kondo, T., Xiong, J. and Golden, S.S. (2003). IdpA encodes an iron-sulfur protein involved in light-dependent modulation of the circadian period in the cyanobacterium Synechococcus elongatus PCC 7942. J. Bacteriol. 185, 1415-22.

Kippert, F. (1991). Essential clock proteins/circadian rhythms in prokaryotes-what is the evidence? Bot. Acta 104, 2-4.

Kitayama, Y., Iwasaki, H., Nishiwaki, T. and Kondo, T. (2003). KaiB functions as an attenuator of KaiC phosphorylation in the cyanobacterial circadian clock system. EMBO J. 22, 1-8

Kondo, T., Strayer, C. A., Kulkarni, R. D., Taylor, W., Ishiura, M., Golden, S. S. and Johnson, C. H. (1993). Circadian rhythms in prokaryotes: luciferase as a reporter of circadian gene expression in cyanobacteria. Proc. Natl. Acad. Sci. USA 90, 5672-5676.

Kondo, T. and Ishiura, M. (1994). Circadian rhythms of cyanobacteria: monitoring the biological clocks of individual colonies by bioluminescence. J. Bacteriol. 176, 1881-1885.

Kondo, T., Tsinoremas, N. F., Golden, S. S., Johnson, C. H., Kutsuna, S. and Ishiura, M. (1994a). Circadian clock mutants of cyanobacteria. Science 266, 1233-1236.

Kondo, T., Ishiura, M., Golden, S.S. and Johnson, C.H. (1994b). Circadian rhythms of cyanobacteria expressed from a luciferase reporter gene. In: Evolution of Circadian Clock. T. Hiroshige and K. Honma, eds. Hokkaido University Press, Sapporo. p. 59-73.

Kondo, T., Mori, T., Lebedeva, N.V., Aoki, S., Ishiura, M., Golden, S.S. (1997). Circadian rhythms in rapidly dividing cyanobacteria. Science 275, 224-227.

Leipe, D.D., Aravind, L., Grishin, N.V. and Koonin, E.V. (2000). The bacterial replicative helicase DnaB evolved from a RecA duplication. Genome Res. 10, 5-16.

Lin, R.-F., Chou, H.-M., Huang, T.-C. (1999). Priority of light/dark entrainment over temperature in setting the 
circadian rhythms of the prokaryote Synechococcus RF-1. Planta 209, 202-206.

Liu, Y., Tsinoremas, N.F., Johnson, C.H., Lebedeva, N.V., Golden, S.S., Ishiura, M. and Kondo, T. (1995). Circadian orchestration of gene expression in cyanobacteria. Genes and Dev. 9, 1469-1478.

Liu, Y., Tsinoremas, N.F., Golden, S.S., Kondo, T. and Johnson, C.H. (1996). Circadian expression of genes involved in the purine biosynthetic pathway of the cyanobacterium Synechococcus sp. strain PCC 7942. Mole. Microbiol. 20, 1071-1081.

Lorne, J., Scheffer, J., Lee, A., Painter, M. and Miao, V.P. (2000). Genes controlling circadian rhythm are widely distributed in cyanobacteria. FEMS Microbiol Lett. 189, 129-133.

Menzel, R. and Gellert M. (1987). Modulation of transcription by DNA supercoiling: a deletion analysis of the Escherichia coli gyrA and gurB promoters. Proc Natl Acad Sci USA 84, 4185-4189.

Min, H., Golden, S.S. (2000). A new circadian Class 2 gene, opcA, whose product is important for reductant production at night in Synechococcus elongatus PCC 7942. J Bacteriol 182, 6214-6221.

Mitsui, A., Kumazawa, S., Takahashi, A., Ikemoto, H., and Arai, T. (1986). Strategy by which nitrogen-fixing unicellular cyanobacteria grow photoautotrophically. Nature 323, 720-722.

Mori, T., Binder, B., Johnson ,C.H. (1996). Circadian gating of cell division in cyanobacteria growing with average doubling times of less than 24 hours. Proc Natl Acad Sci USA 93, 10183-10188.

Mori, T. and Johnson, C.H. (2000). Circadian control of cell division in unicellular organisms. In: Progress in Cell Cycle Research, Volume 4. L. Meijer, A. Jezequel, and B. Ducommun, eds. Kluwer Academic/Plenum Press, N.Y., p. 185-192.

Mori, T, and Johnson, C.H. (2001a). Independence of circadian timing from cell division in cyanobacteria. $J$. Bacteriol. 183, 2439-2444.

Mori, T. and Johnson, C.H. (2001b). Circadian programming in cyanobacteria. Sem. Cell Develop. Biol. 12, 271-278.

Mori, T., Saveliev, S.V., Xu, Y., Stafford, W.F., Cox, M.M., Inman, R.B., and Johnson, C.H. (2002). Circadian clock protein KaiC forms ATP-dependent hexameric rings and binds DNA. Proc. Natl. Acad. Sci. USA 99, 1720317208.

Mutsuda, M., Michel, K.P., Zhang, X., Montgomery, B.L. and Golden, S.S. (2003). Biochemical properties of CikA, an unusual phytochrome-like histidine protein kinase that resets the circadian clock in Synechococcus elongatus PCC 7942. J. Biol. Chem. 278, 19102-19110.

Nair, U., Ditty, J.L., Min, H., and Golden, S.S. (2002). Roles for sigma factors in global circadian regulation of the cyanobacterial genome. J. Bacteriol. 184, 3530-3538.
Nishimura, H., Nakahira, Y., Imai, K., Tsuruhara, A., Kondo, H., Hayashi, H., Hirai, M., Saito, H. and Kondo, T. (2002). Mutations in KaiA, a clock protein, extend the period of circadian rhythm in the cyanobacterium Synechococcus elongatus PCC 7942. Microbiol. 148, 2903-2909.

Nishiwaki, T., Iwasaki, H., Ishiura, M. and Kondo, T. (2000). Nucleotide binding and autophosphorylation of the clock protein KaiC as a circadian timing process of cyanobacteria. Proc. Natl. Acad. Sci. USA 97, 495-499.

Ouyang, Y., Andersson, C.R., Kondo, T., Golden, S.S. and Johnson, C.H. (1998). Resonating circadian clocks enhance fitness in cyanobacteria. Proc. Natl. Acad. Sci. USA 95, 8660-8664.

Pettijohn, D. (1976). Prokaryotic DNA in nucleiod structure. CRC Critical Rev. Biochem. 4, 175-202.

Pittendrigh, C.S. (1993). Temporal organization: reflections of a Darwinian clock-watcher. Annu. Rev. Physiol. 55, 17-54.

Pruss, G.J. and Drlica, K. (1989). DNA supercoiling and prokaryotic transcription. Cell 56, 521-523.

Salvador, M.L., Klein, U. and Bogorad, L. (1998). Endogenous fluctuations of DNA topology in the chloroplast of Chlamydomonas reinhardtii. Mole. Cell Biol. 18, 7235-7242.

Schmitz, O., Katayama, M., Williams, S.B., Kondo, T. and Golden, S.S. (2000). CikA, a bacteriophytochrome that resets the cyanobacterial circadian clock. Science 289 , 765-768.

Taniguchi, Y., Yamaguchi, A., Hijikata, A, Iwasaki, H., Kamagata, K., Ishiura, M., Go, M. and Kondo, T. (2001). Two KaiA-binding domains of cyanobacterial circadian clock protein KaiC. FEBS Letters 496, 86-90.

Trun, N.J. and Marko, J.F. (1998). Architecture of a bacterial chromosome. ASM News 64, 276-283.

Tsinoremas, N.F., Ishiura, M., Kondo, T., Andersson, C.R., Tanaka, K., Takahashi, H., Johnson, C.H. and Golden, S.S. (1996). A sigma factor that modifies the circadian expression of a subset of genes in cyanobacteria. EMBO Journal 15, 2488-2495.

Williams, S.B., Vakonakis, I., Golden, S.S., and LiWang, A.C. (2002). Structure and function from the circadian clock protein KaiA of Synechococcus elongatus: a potential clock input mechanism. Proc. Natl. Acad. Sci. USA 99, 15357-15362.

Xu, Y., Piston, D. and C.H. Johnson. (1999). A bioluminescence resonance energy transfer (BRET) system: Application to interacting circadian clock proteins. Proc. Natl. Acad. Sci. USA 96, 151-156.

Xu, Y., Mori, T. and Johnson, C.H. (2000). Circadian clockprotein expression in cyanobacteria: rhythms and phasesetting. EMBO J. 19, 3349-57.

Xu, Y., Mori, T. and Johnson, C.H. (2003). Cyanobacterial circadian clockwork: roles of KaiA, KaiB, and the kaiBC promoter in regulating KaiC. EMBO J. 22, 2117-2126. 


\section{Further Reading}

Caister Academic Press is a leading academic publisher of advanced texts in microbiology, molecular biology and medical research. Full details of all our publications at caister.com

- MALDI-TOF Mass Spectrometry in Microbiology Edited by: M Kostrzewa, S Schubert (2016) www.caister.com/malditof

- Aspergillus and Penicillium in the Post-genomic Era Edited by: RP Vries, IB Gelber, MR Andersen (2016) www.caister.com/aspergillus2

- The Bacteriocins: Current Knowledge and Future Prospects Edited by: RL Dorit, SM Roy, MA Riley (2016)

www.caister.com/bacteriocins

- Omics in Plant Disease Resistance Edited by: V Bhadauria (2016) www.caister.com/opd

- Acidophiles: Life in Extremely Acidic Environments Edited by: R Quatrini, DB Johnson (2016) www.caister.com/acidophiles

- Climate Change and Microbial Ecology: Current Research and Future Trend

Edited by: J Marxsen (2016)

www.caister.com/climate

- Biofilms in Bioremediation: Current Research and Emerging Technologies

Edited by: G Lear (2016)

www.caister.com/biorem

- Microalgae: Current Research and Applications Edited by: MN Tsaloglou (2016) www.caister.com/microalgae

- Gas Plasma Sterilization in Microbiology: Theory, Applications, Pitfalls and New Perspectives Edited by: H Shintani, A Sakudo (2016) www.caister.com/gasplasma

- Virus Evolution: Current Research and Future Directions Edited by: SC Weaver, M Denison, M Roossinck, et al. (2016) www.caister.com/virusevol

- Arboviruses: Molecular Biology, Evolution and Control Edited by: N Vasilakis, DJ Gubler (2016) www.caister.com/arbo

- Shigella: Molecular and Cellular Biology Edited by: WD Picking, WL Picking (2016) www.caister.com/shigella

-Aquatic Biofilms: Ecology, Water Quality and Wastewater Treatment

Edited by: AM Romaní, H Guasch, MD Balaguer (2016)

www.caister.com/aquaticbiofilms

- Alphaviruses: Current Biology

Edited by: S Mahalingam, L Herrero, B Herring (2016)

www.caister.com/alpha

- Thermophilic Microorganisms

Edited by: F Li (2015)

www.caister.com/thermophile
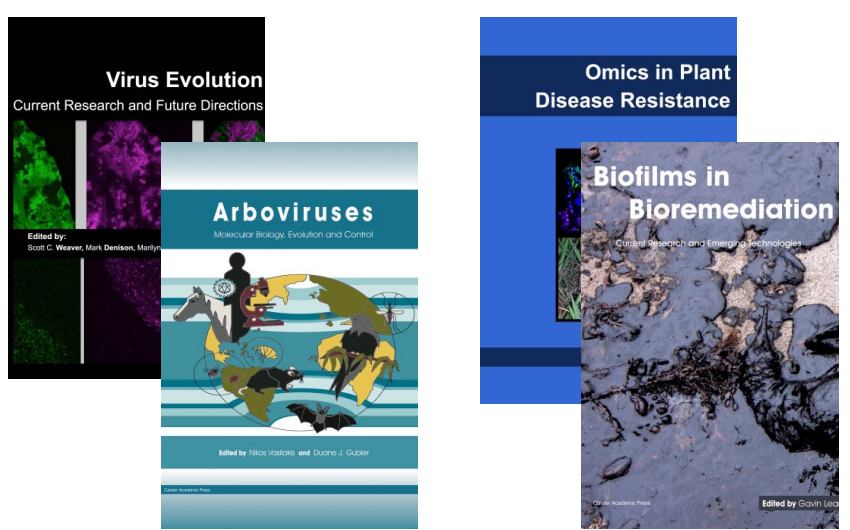
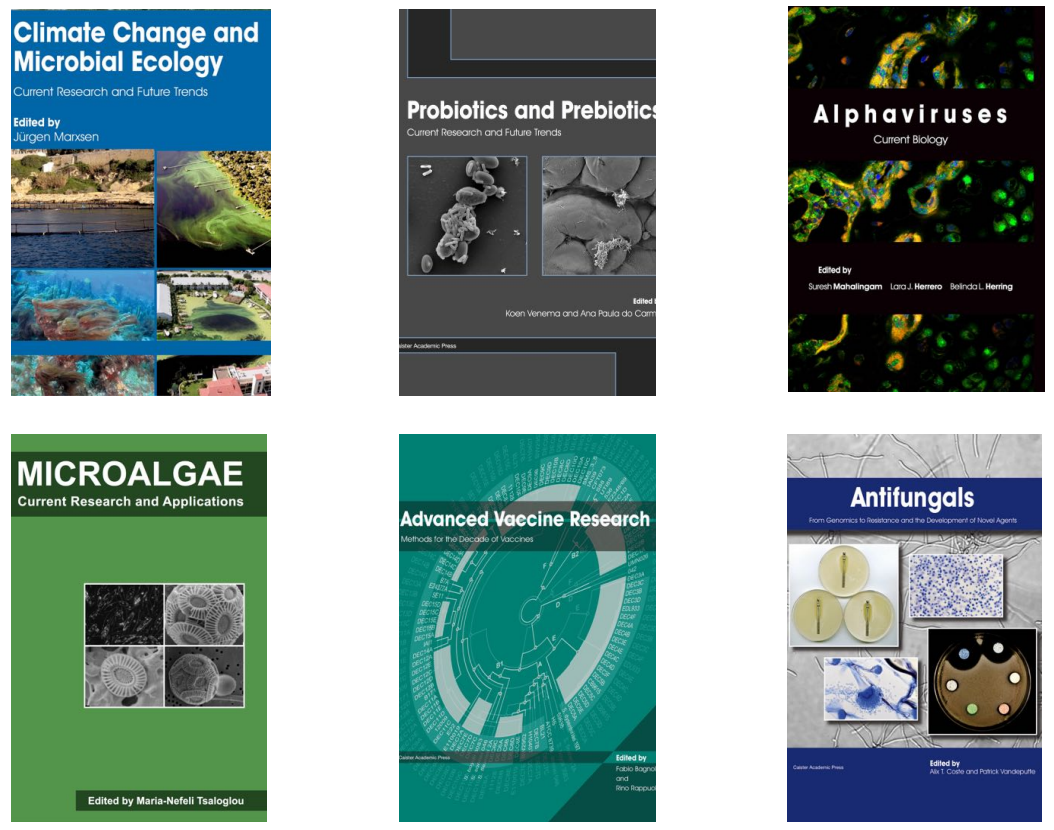

- Flow Cytometry in Microbiology: Technology and Applications Edited by: MG Wilkinson (2015) www.caister.com/flow

- Probiotics and Prebiotics: Current Research and Future Trends Edited by: K Venema, AP Carmo (2015) www.caister.com/probiotics

- Epigenetics: Current Research and Emerging Trends Edited by: BP Chadwick (2015) www.caister.com/epigenetics2015

- Corynebacterium glutamicum: From Systems Biology to Biotechnological Applications

Edited by: A Burkovski (2015)

www.caister.com/cory2

- Advanced Vaccine Research Methods for the Decade of Vaccines

Edited by: F Bagnoli, R Rappuoli (2015)

www.caister.com/vaccines

- Antifungals: From Genomics to Resistance and the Development of Novel Agents

Edited by: AT Coste, P Vandeputte (2015)

www.caister.com/antifungals

- Bacteria-Plant Interactions: Advanced Research and Future Trends Edited by: J Murillo, BA Vinatzer, RW Jackson, et al. (2015) www.caister.com/bacteria-plant

\section{- Aeromonas}

Edited by: J Graf (2015)

www.caister.com/aeromonas

- Antibiotics: Current Innovations and Future Trends

Edited by: S Sánchez, AL Demain (2015)

www.caister.com/antibiotics

- Leishmania: Current Biology and Contro Edited by: S Adak, R Datta (2015) www.caister.com/leish2

- Acanthamoeba: Biology and Pathogenesis (2nd edition) Author: NA Khan (2015)

www.caister.com/acanthamoeba2

- Microarrays: Current Technology, Innovations and Applications Edited by: Z He (2014)

www.caister.com/microarrays2

- Metagenomics of the Microbial Nitrogen Cycle: Theory, Methods and Applications

Edited by: D Marco (2014)

www.caister.com/n2 